\title{
Multi-toll-type motorway stations optimal layout
}

\author{
A. Pratelli ${ }^{1} \&$ F. Schoen ${ }^{2}$ \\ ${ }^{1}$ Dipartimento di Ingegneria Civile, University of Pisa, Italy \\ ${ }^{2}$ Dipartimento di Sistemi e Informatica, University of Florence, Italy
}

\begin{abstract}
The objective of this paper is to develop a toll station layout optimisation methodology based on toll plaza circulation safety analysis. Then we propose a mathematical model assessing the expected level of traffic safety associated with the toll station layout, actual or planned, due to a given configuration for toll gate types and positions. The model has been conceived in order to let the planner be able to decide the proper sequence of gate types which minimises the risk of accidents. The decision variables are mainly related to the assignment of a gate type - manual gate with operator, ETC gate, semiautomatic or short stop gate to each gate position in the station. The resulting multi-toll-type assignment model, or MTTA, takes into account the probability of interaction between pairs of traffic streams originated from the different lanes of the highway and directed to specific gate types; different severity and risk indices are associated both with vehicle types and with destination gate types. The computational tests have been performed on observed data collected during several hours of video-camera images recorded in five different motorway multi-type toll plazas located in Tuscany (Italy). Our results support the evidence that gates associated to higher risk traffic flows, e.g. traffic directed to ETC gates which approaches the toll station at speeds significantly above the average, should be locate in a central position with respect to other gate types. Moreover, the experimental results disagree with the general operations practice of many Italian motorway Agencies, which usually place ETC gates on the far side of the toll plaza.

Keywords: motorway toll station design, multi-toll-type collection, weaving accident risk, electronic toll collection, quadratic assignment.
\end{abstract}




\section{Introduction}

A multi-type toll motorway station consists in a set of different types of tollgates, and involves two different user-behaviours and as many toll lanes: stop way lane (such as gate with operator, smart card and/or credit card gate - in Italy named "Viacard" gate - self service gate with small change) and no-stop way lane (such as automatic vehicle identification, AVI, or electronic toll collection, ETC, gate - in Italy known as "Telepass" gate).

Vehicles approaching a toll plaza are frequently involved in accidents, mostly due to sudden lane changes of vehicle drivers who try to join the shortest queue, or, in addition, due to the interactions between vehicles directed to distinct types of available tollgates. Reciprocal position of each type of tollgate is crucial for safety goals. Trajectories of higher speed vehicles directed to ETC gates need to be managed in respect to lower speeds of vehicles approaching any other type of stop gate, i.e. manual gate and smart card or semiautomatic gate. Determination both of the plaza size and of the proper gate type sequence, i.e. toll station layout, in the actual planning process is quite important for an optimal design in a safer sense - of the toll station and essential to its future operational efficiency.

In spite of the relevance of the matter, relatively little research has been directed on motorway toll stations design in the past. Last years view a general growing interest on planning and design issues, mainly due to large diffusion of the ETC technologies. Some research works have reviewed the operations of toll plazas in order to determine the optimal number of tollgates staffing during specific times of the day. The goal in this type of analysis is to increase or decrease staffing to match peak and off-peak demand. Other studies deal with methodologies for level-of-service (LOS) analysis of toll plazas.

In the early 1950s Edie [3] presented his pioneer study on operating characteristics of New York Authority tollgates. The paper presented an extensive analysis on queue lengths and concluded with some insights on personnel scheduling.

Kopanezou and co-workers [8] conducted research on queuing at toll gates through computer program simulating driver behaviour and enabling system analysis and design relationships. The work of Gulewicz and Danko [7] was based on a large field database from the eight-lane toll plaza with two arrival lanes at the Outerbridge Crossing in New York. They pointed out the following four general driving habits: 1) most driver enter a toll lane on the same side of the toll plaza from which they exited the span; 2) most drivers, once they have selected which half of the plaza to enter, select the tollgate with the shortest queue on that side; 3 ) some drivers were observed entering the tollgate with the shortest queue, even though an empty gate was available (it was believed that these drivers focused on only the other vehicles in the plaza and not on the signals above the tollgates, this caused them to believe that the empty gates were closed); 4) a small percentage of motorists appeared to choose a tollgate randomly. The driving habits just quoted above have been also observed in some Italian motorway toll plazas [12] analysing field data that confirm that drivers 
prefer to use a tollgate that does not require lane changes. Only if the number of queuing vehicles is a three or more car, then an arriving driver will change lane and go to a gate with a shorter queue. Polus $[10,11]$ presented the prominent consideration in conducting an analysis of an urban toll-plaza with a simulation model. His work pointed out that the main parameters influencing the design process are the throughput distribution of the various gates, the through-volume, the percentage of trucks, the proportion of manual gate users and the percentage of ETC equipped users. Analysis of toll stations with different types of toll collection gates has been approached by microscopic traffic simulation by Astarita and co-workers [2]. Their model is based on a set of car-following and lane-changing rules, and it seems to be also able to take into account the instance with mixed tollgate type composition.

This paper describes a mathematical model assessing the expected level of traffic safety associated with the toll station layout, actual or planned, due to a given configuration for gate types and positions. The model takes into account the probability of interaction between pairs of traffic streams originated from the different lanes of the highway and directed to specific gate types. Our model has been conceived in order to let the planner be able to decide the proper sequence of gate types that minimises the risk of accidents. The paper is closed discussing the computational results obtained from the model application to a real database.

\section{The Multi-Toll-Type Assignment model}

Our model can be referred as the Multi-Toll-Type Assignment problem, or MTTA problem [13]. It has been conceived in order to let the planner be able to decide the proper sequence of gate types that minimises the risk of accidents. The decision variables $\delta(g, t)$ are mainly related to the assignment of a gate type $t$ - i.e. stop gate (manual gate with operator, semiautomatic self-service or credit card operated) or no-stop gate (ETC gate) or mixed stop no-stop gate (e.g. both self- service and ETC operated) - to each gate position $g$ in the station. In particular, let $\delta(g, t)=1$ if tollgate $g$ (with $g=1 \ldots N$ ) is assigned type $t$ (with $t$ equal either to stop, $t=S t o p$, or no-stop, $t=N o S t o p$, or mixed, $t=$ Mix.

The model takes into account the probability of interaction between pairs of traffic streams originated from the different lanes of the highway and directed to specific gate types.

Let us now assume that a toll station with a total of $N$ gates has to be designed, and that decisions on the characteristics of each gate have been already taken by the motorway agency. Let: $N_{\text {NoStop }}$ gates totally electronic, or ETC (e.g. "Telepass") gates; $N_{\text {Stop }}$ gates with credit card payment (e.g. Viacard) or self-service with exact change or manually attended with operator; $N_{\text {Mix }}$ mixed gates, available both for ETC and non-ETC equipped vehicles. Then we have:

$$
N=N_{\text {Stop }}+N_{\text {NoStop }}+N_{\text {Mix }}
$$

The planners have to choose in which sequence these $N$ gates have to be placed, bearing in his mind that drivers arriving from different lanes, directed towards 
different gates will incur different risk levels of accident. Accident risk in a toll station is related to the probability of the occurrence of interference between two vehicles moving at the same time across the toll plaza. One can assume that the tollgate type influences the risk of an accident, as cars directed towards an automatic gate will approach the plaza at a relatively higher speed than cars directed towards manual or semiautomatic gates.

We set up a risk objective function defined in the following way: let two gates $i$ and $j$ be considered, with $i<j$ assuming that gates are numbered from left to right. An accident might occur between traffic approaching the toll plaza arriving from the normal lane directed to gate $i$ and flow arriving from the other lane, directed towards gate $j$.

Then a risk factor is associated to:

- gate factor, GF: characteristics of the three respective tollgate types (either ETC, type "NoStop", or manual and/or credit card and/or self-service, type "Stop", or mixed, type "Mix");

- vehicle factor, $V F$ : each pair of approaching lanes:

- lane factor, $L F$ : each possible combination of traffic types in both approaching lanes (either cars or trucks);

- traffic composition, TC: percentage of passenger cars (percentage heavy occupancy vehicles) using a tollgate of a given type.

- reciprocal distance factor, $D F(|i, j|)$ : distance between the involved gate $i$ and gate $j$ (greater distance implies higher risk).

Let $|i-j|$ be the reciprocal distance between the involved gates, then $D F(|i, j|)$ factor is assumed according to [13]:

$$
D F(|i, j|)=1+\frac{|i-j|}{5 N}
$$

The global risk of track weaving of each pair of vehicles in a toll plaza of $N$ toll gates of various types and fixed placement is given by the summation of the risk associate to each possible instance due by type of involved tollgates, their reciprocal positions, characteristics of arriving vehicles and their respective approaching lane. If all interference conditions are assumed to be independent, the product of probability of single events gives the probability of the occurrence of an accident. Then the above risk factors multiply each other.

For example, the risk associated to a car from the normal lane directed to gate $i$ (ETC, or "NoStop" type) crossing a car from the overpass lane directed to gate $j$ (credit card, or "Stop" type) is given by:

$$
L F(\text { normal }) L F(\text { overtake }) G F(\text { NoStop; Stop }) V F(\text { car } ; \text { car }) D F(|i-j|)
$$

The resulting objective function combines different risk factors, which are associated to vehicle types, destination gate types and traffic composition. One obtains a quadratic assignment problem in which binary decision variables characterise the association between physical gate place and tollgate type. From a theoretical point of view, one can write: 


$$
\min \sum_{g_{i}, g_{j} \in 1 \ldots N} \operatorname{risk}\left(g_{i}, t_{i}, g_{j}, t_{j}\right) \delta\left(g_{i}, t_{i}\right) \delta\left(g_{j}, t_{j}\right)
$$

Constraint (3) is added in order to be sure that each tollgate has associated to a specified type (among Stop, NoStop and Mix). Constraints (4), (5) and (6) state that the designed number of tollgates of each type exactly composes the whole station:

$$
\begin{gathered}
\sum_{t \in\{\text { Stop }, \text { NoStop }, \text { Mix }\}} \delta(g, t)=1 \quad \forall g \in 1 \ldots . . N \\
\sum_{g \in 1 \ldots N} \delta(g, S)=N_{\text {Stop }} \\
\sum_{g \in 1 \ldots N} \delta(g, N S)=N_{\text {NoStop }} \\
\sum_{g \in 1 \ldots N} \delta(g, M)=N_{\text {Mix }}
\end{gathered}
$$

It is well known that quadratic assignment problems are extremely hard and computational burdensome, if not impossible, to solve [9]. Nevertheless, because practical design instances reach a maximum of $18 \div 20$ tollgates, we are able to obtain optimal solutions even for large sized toll stations in a few minutes on a normal personal computer.

\section{Model calibrations}

The model calibrations require the values of four of five parameters appearing in the risk function basic structure. They are $T C, L F, G F$ and $V F$, while the fifth parameter $D F$ is a geometric data. Motorway toll stations have been divided into three general classes: small, with no more five gates $(N \leq 5)$; medium, $6 \leq N \leq 10$; large, $N \geq 11$.

Following this classification we have recorded, both in three small sized motorway stations and in two medium sized ones, twelve hours of camera films of random sampling data related to different periods, each ranging from 10 a.m. to 14 p.m of a weekday. All the observed toll stations belong to the network operated by SALT p.a. motorway agency in Western Tuscany (IT) and they are: three small - namely A12-Lucca Ovest LU/VG, A11-Lucca Nord VG/LU and A12-Livorno FI/GE - the first with 3 tollgates and the remainders with 3 tollgates; two medium - namely A11-Lucca Nord LU/VG and A12-Livorno GE/FI - with 9 and 8 tollgates, respectively.

The analysis of experimental data gives the values of $T C$ as observed frequencies, or percentages, which are resumed in the following both for small stations, and for medium stations. One can observe that the small stations (see Table 1) show a 50/50 distribution of passenger cars among Stop and NoStop gates. Moreover, there is a high percentage $(70 \%)$ of heavy vehicles using ETC gates. Medium sized stations (see Table 2) show growing gaps in user 
proportions of Stop and NoStop gates. While user percentages of mixed gates are substantially equal for both station sizes.

Table 1: $\quad$ Estimated values for $T C$ factor in small stations.

\begin{tabular}{|l|c|c|c|}
\hline$T C$ & Stop & NoStop & Mixed \\
\hline Passenger cars & 0.40 & 0.39 & 0.21 \\
\hline Heavy vehicles & 0.13 & 0.70 & 0.17 \\
\hline
\end{tabular}

Table 2: $\quad$ Estimated values for TC factor in medium stations.

\begin{tabular}{|l|c|c|c|}
\hline TC & Stop & NoStop & Mixed \\
\hline Passenger cars & 0.51 & 0.31 & 0.18 \\
\hline Heavy vehicles & 0.24 & 0.57 & 0.19 \\
\hline
\end{tabular}

The calibration process of $L F$ factor has been conducted in respect to standard calibration procedures for Poisson distributed random variables [15]. So it is well known that there is a probability to observe $x$ vehicles travelling through any tollgate during time $t$ when $\lambda$ is the average flow given by:

$$
P_{x}=\frac{e^{-\lambda t} \cdot(\lambda t)^{x}}{x !}
$$

In particular, the lane factor $L F$ is related to a pair of vehicles and requires the analysis, in term of observed frequencies, of the following simple events: a) one vehicle travels on the normal lane; b) other vehicle must travel on the overpass lane; c) the vehicle travelling on the normal lane is directed to any tollgate $i$;) the other vehicle on the overpass lane must be directed to any tollgate $j$, where $j>i$ holds. Each one of these simple events is disjoint and independent. The happening probability of one of them, given the others, is a conditional probability given by the product of each simple event probability. This last is estimated from the observed data in term of relative frequency. The hypothesis of independence is admissible for all five observed fields because data are gathered under traffic flow conditions always below $500 \mathrm{veic} / \mathrm{h}$ [14].

Two vehicles, one exiting from the normal lane and the other from the overpass lane, should intersect their tracks only and only if at the same time $t$ the first vehicle approaches any gate $i$ and the second vehicle approaches any gate $j$ successive to previous gate $i$ (i.e. $j>i$ ). The related probability becomes:

$$
P_{\text {wav }}=\frac{n_{\text {nor }}}{n} \cdot \frac{e^{-\lambda_{i} t_{i}}\left(\lambda_{i} t_{i}\right)^{x}}{x !} \cdot \frac{n_{\text {ovp }}}{n} \cdot \frac{e^{-\lambda_{j} t_{i}}\left(\lambda_{j} t_{i}\right)^{x}}{x !}
$$

Finally, the weaving probability during a time $t_{j}$ for a pair of vehicles both approaching the toll plaza of a station with $N$ tollgates, one coming from the normal lane and the other from the overpass lane, is expressed by: 


$$
\begin{gathered}
P_{\text {wav }, \text { nor }}=\left[\sum_{i=1}^{N-1} \frac{n_{\text {nor }}}{n} \cdot \frac{e^{-\lambda_{i} t_{i}}\left(\lambda_{i} t_{i}\right)^{x}}{x !} \cdot \sum_{j=i+1}^{N}\left[\frac{n_{\text {ovp }}}{n} \cdot \frac{e^{-\lambda_{j} t_{i}}\left(\lambda_{j} t_{i}\right)^{x}}{x !}\right]\right] \\
P_{\text {wav }, \text { ovp }}=\left[\sum_{j=i+1}^{N} \frac{n_{\text {ovp }}}{n} \cdot \frac{e^{-\lambda_{j} t_{j}}\left(\lambda_{j} t_{j}\right)^{x}}{x !} \cdot \sum_{i=1}^{N-1}\left[\frac{n_{\text {nor }}}{n} \cdot \frac{e^{-\lambda_{i} t_{j}}\left(\lambda_{i} t_{j}\right)^{x}}{x !}\right]\right]
\end{gathered}
$$

where: $n_{\text {nor }} / n$ and $n_{\text {ovp }} / n$ are the (frequencies) probabilities to observe a vehicle exiting from the normal lane or from the overpass lane, respectively; $\lambda_{i}$ and $\lambda_{j}$ is the average flow traversing gates $i$ or gate $j$, respectively. The related $L F$ factor estimates are obtained by substituting the observed values in (11) and (10).

Table 3: $\quad$ Lane factor calibrated values.

\begin{tabular}{|l|c|c|}
\hline$L F$ & Normal lane & Overpass lane \\
\hline Small stations & 1.0 & 1.1 \\
\hline Medium stations & 1.7 & 1.0 \\
\hline
\end{tabular}

Table 4: $\quad$ Vehicle factor calibrated values.

\begin{tabular}{|l|l|c|c|}
\hline \multicolumn{2}{|c|}{$V F$} & Cars & Heavy \\
\hline \multirow{3}{*}{ Small stations } & Cars & 7.5 & 5.2 \\
\cline { 2 - 4 } & Heavy & 5.2 & 1.0 \\
\hline \multirow{3}{*}{ Medium stations } & Cars & 4.0 & 5.2 \\
\cline { 2 - 4 } & Heavy & 5.2 & 1.0 \\
\hline
\end{tabular}

Table 5: $\quad$ Gate factor calibrated values.

\begin{tabular}{|l|l|c|c|c|}
\hline \multicolumn{2}{|c|}{$G F$} & Stop & NoStop & Mixed \\
\hline \multirow{3}{*}{ Small stations } & Stop & 1.0 & 1.5 & 1.0 \\
\cline { 2 - 5 } & NoStop & 1.5 & 1.0 & 1.2 \\
\cline { 2 - 5 } & Mixed & 1.0 & 1.2 & 1.0 \\
\hline \multirow{3}{*}{ Medium stations } & Stop & 1.0 & 6.3 & 1.0 \\
\cline { 2 - 5 } & NoStop & 6.3 & 1.0 & 12.3 \\
\cline { 2 - 5 } & Mixed & 1.0 & 12.3 & 1.0 \\
\hline
\end{tabular}

The calibration processes of remaining $G F$ and $V F$ factors followed similar procedures as the above [14]. Finally, all different factor values (say $a_{i}$ ) have been standardized in respect their minimum (say $a_{\text {min }}$ ) by:

$$
y_{i}=\frac{a_{i}}{a_{\min }}
$$

The final values given by (11) are reported in Table 3 to Table 5 . 


\section{Applications}

The calibrated model was applied to the toll plazas quoted above for data observation. In particular, we considered both the actual traffic and a doubled value. Moreover, the heavy vehicle percentage has been varied in order to appraise its influence. Three toll station compositions have been examined: actual; minimum risk, or optimal; maximum risk. Then model applications have involved the computation of a set of risk level values related to many combinations both of traffic flows and toll station layouts.

For the sake of brevity, here it is reported a resume of whole calculus and are highlighted only the main results. This lead to Fig. 1 that depicts both the actual and the resultant layouts for A12-Lucca Ovest LU/VG respect to actual traffic flows. The same contents are depicted in Fig. 2 related to A12-Livorno GE/FI.

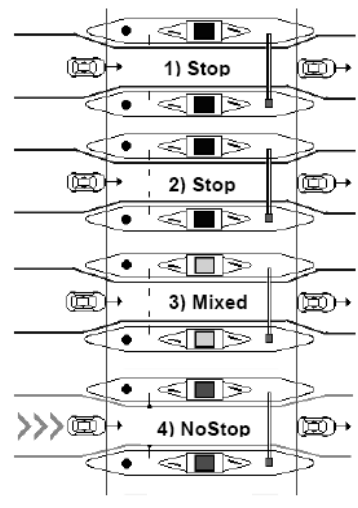

(a)

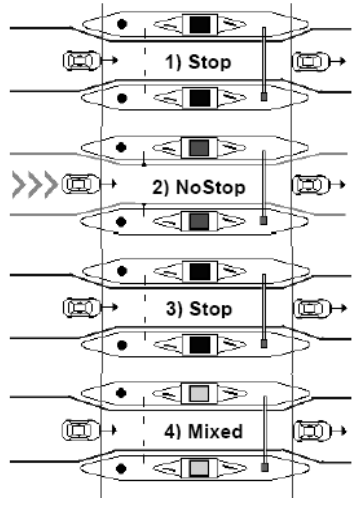

(b)

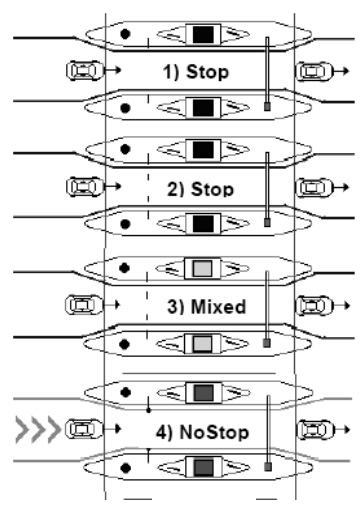

(c)

Figure 1: Toll station A12-LuccaOvest LU/VG: (a) actual layout; (b) minimum risk layout; (c) maximum risk layout.

Figure 1 is referred to a small station ( $N \leq 5$ gates) and one can see that the actual layout is equal to the maximum risk layout resulting in model application, where the NoStop gate is placed at the right extreme.

Figure 2 is referred to a medium station $(6 \leq N \leq 10)$ and the minimum risk layout shows the NoStop gate and the Mixed gate (i.e. a quasi-NoStop gate) placed in the middle. On the contrary, these two gates result placed on both opposite extremes in the minimum risk solution.

Simulations with doubled traffic flows show resulting layouts quite similar to the previous ones. Moreover, changing in flow composition due to growing percentages of heavy vehicles have shown as many growing values of risk for any given layout. 


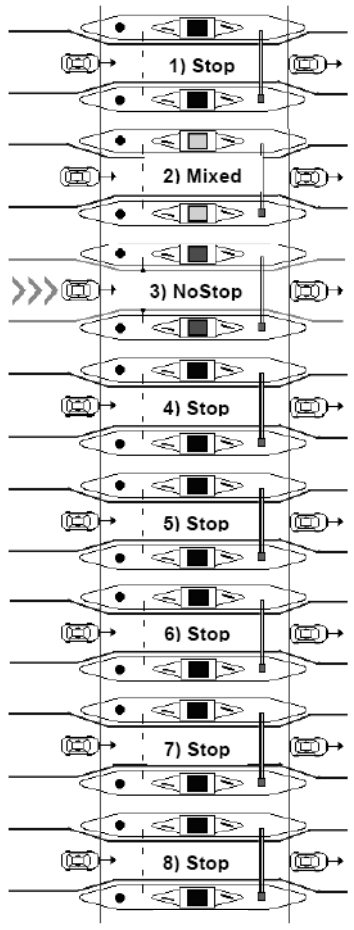

(a)

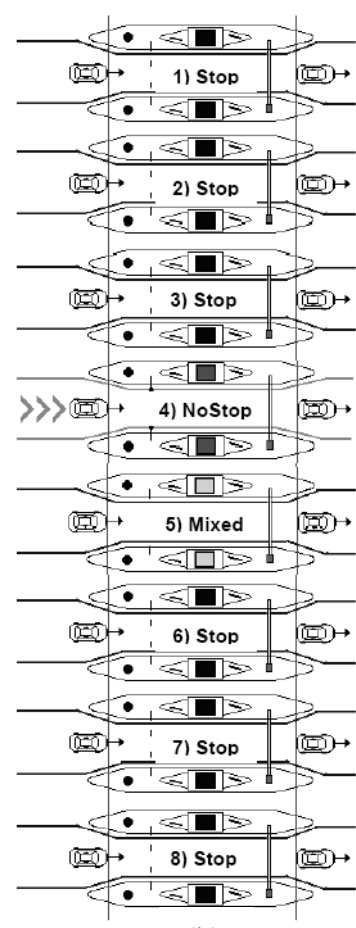

(b)

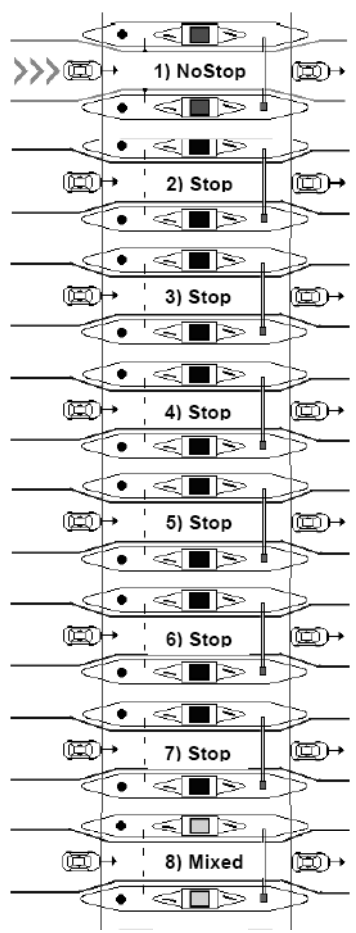

(c)

Figure 2: Toll station A12-Livorno GE/FI: (a) actual layout; (b) minimum risk layout; (c) maximum risk layout.

\section{Conclusions}

The proposed MTTA model belongs to the family of mathematical programming approaches for the optimisation design of toll plazas. Its main original feature in respect to previous models, e.g. $[2,7,8]$, is the capability to explicitly consider a set of circulation safety related factors as basic design inputs. The model has been calibrated with experimental data collected processing several hours of video-camera images recorded in five different motorway toll plazas located in Tuscany (Italy). Computational experiments show that gates associated to higher risk traffic flows, e.g. traffic directed to ETC, or "Telepass", gates which approaches the toll station at speeds significantly above the average, should be locate in a central position with respect to other gate types. Our experimental results disagree with the general empiric operations practice of placing the ETC gates on the extreme sides of the toll plaza.

The Italian motorway toll network is $5.584 \mathrm{~km}$ length, with 459 toll stations for 3.600 toll gates, among them 1.471 of "Telepass" type and over 2.100.000 vehicles are equipped with ETC transponder for electronic toll [4]. The 
percentage of ETC equipped vehicles raised from $15 \%$ in 1997 to $38 \%$ in 2000 , with over 57 millions of transits on "Telepass" gates recorded during year 2000 [6]. For the same year 2000, statistics show that there have been 38.705 accidents on the whole Italian motorway network [16]. Even if it is not specified, one can properly think that some of these accidents happened in toll plazas. At the same time, one can properly ask to himself if any design safety based mathematical criterion for motorway toll station layouts should be better than mere empiricism.

\section{Acknowledgement}

We herewith wish to thank SALT p.a., Lido di Camaiore (Italy) who, by allowing us the access to its motorway stations, have strongly helped our research efforts.

\section{References}

[1] AISCAT, Notes about toll motorway and toll system in Italy, Internal Report n. 95004, Rome 1994.

[2] Astarita V., Florian M. and Musolino G., A microscopic traffic simulation model for the evaluation of toll station systems, Proceedings of the IEEE 2001, Oakland, pp. 694-699, 2001

[3] Edie L.C., Traffic delays at toll booths, Journal of the Operations Research Society of America vol. 2, pp. 107-138, 1954.

[4] Cialone E., Le autostrade in Europa, Autostrade 4, pp. 45-71, 2002.

[5] Fourer R., Gay D.M. and Kerninghan B.W., AMPL: a modelling language for mathematical programming, Boyd \& Fraser, Danvers, Mass., 1993.

[6] Giorgi P. e Battiboia S., Telepass: l'evoluzione della specie, Autostrade1, pp. 3-23, 2001.

[7] Gulewicz V. and Danko J., Simulation-based approach to evaluating optimal lane staffing requirements for toll plazas, Transp. Res. Record 1484, pp. 33-39, 1994.

[8] Kopanezou E., Lambropoulos S. and Manolopoulos N., Toll booth management via simulation, Proceedings of the 4th Int. Conf. on Civil and Structural Engineering Computing, pp. 275-284, 1989.

[9] Luenberger D.G., Linear and nonlinear programming, Addison Wesley, Reading, Mass. 1984.

[10] Polus A., Methodology and simulation of toll-plaza analysis, Road and Transport Research vol. 5, pp. 44-56, 1996.

[11] Polus A., Dynamic equilibrium and concepts of toll-plaza planning, Traffic Engineering and Control, pp. 230-233, 1998.

[12] Pratelli A., De Sio G. e Palavisini A., Indagine sperimentale e tecnologie per l'esazione del pedaggio sulle autostrade, Firenze University Press, Firenze 2001.

[13] Pratelli A. and Schoen F., Optimal design of motorway toll stations, Proc. of the ETC-European Transport Conference, Strasbourg, PTRC, 2003. 
Urban Transport XII: Urban Transport and the Environment in the 21st Century 921

[14] Rocchio R.A., Progetto di una stazione di pedaggio con differenti tipi di porte di esazione, Graduation Thesis a.y. 2003-04, University of Pisa, 2004.

[15] Stuart A., Kendall M. and Ord J.K., The advanced theory of statistics. Griffin, London 1983.

[16] Valori G.E., La situazione generale della rete autostradale italiana al 31 dicembre 2000, Autostrade 2, pp. 4-11, 2001. 